Research Paper

\title{
A Survival Model in Locally Advanced and Metastatic Pancreatic Ductal Adenocarcinoma
}

\author{
Yi Wang1, $2^{*}$, Xiuying Xiao ${ }^{*}$, Tianyi Wang ${ }^{*}$, Lin $\mathrm{Li}^{2}$, Yue Zhu ${ }^{1}$, Haiyan $\mathrm{Xu}^{1}$, Yuening $\mathrm{Chu}^{2}$, Feng Jiao ${ }^{1}$, Jiujie \\ Cui $^{1 凶}$, Liwei Wang ${ }^{1 凶}$ \\ 1. Department of Oncology, State Key Laboratory for Oncogenes and Related Genes, Renji Hospital, School of Medicine, Shanghai Jiaotong University, \\ Shanghai Cancer Institute, Shanghai, China, 200127 \\ 2. Department of Oncology, Shanghai General Hospital, Shanghai Jiao Tong University School of Medicine (originally named "Shanghai First People's \\ Hospital"), Shanghai, China, 201620 \\ *these authors contributed equally to this work.
}

$\square$ Corresponding author: Liwei Wang, Department of Oncology, State Key Laboratory for Oncogenes and Related Genes, Renji Hospital, School of Medicine, Shanghai Jiaotong University, Shanghai Cancer Institute, Shanghai, China, 200127. (Phone: +86-21-68385559, Fax: +86-21-68385559, E-mail: liweiwang@shsmu.edu.cn). Jiujie Cui, Department of Oncology, State Key Laboratory for Oncogenes and Related Genes, Renji Hospital, School of Medicine, Shanghai Jiaotong University, Shanghai Cancer Institute, Shanghai, China, 200127. (Phone: +86-21-68385559, Fax: +86-21-68385559, E-mail: cuijiujie@126.com).

(c) Ivyspring International Publisher. This is an open access article distributed under the terms of the Creative Commons Attribution (CC BY-NC) license (https://creativecommons.org/licenses/by-nc/4.0/). See http://ivyspring.com/terms for full terms and conditions.

Received: 2017.11.23; Accepted: 2018.02.28; Published: 2018.03.22

\begin{abstract}
The prognostic role of serum LDH, CA19-9, CRP and ALB in PDAC patients are controversial. In contrast to single factor, there is much less information about the prognostic value of the combination of the four factors in locally advanced and metastatic PDAC patients. It's essential to set up a survival model with the combination of tumor metabolism, tumor biomarker, systemic inflammation and nutritional status to eliminate the prognostic inaccuracy in single biomarker. 94 advanced PDAC patients who received palliative chemotherapy from 2009 to 2017 were recruited for this study. The predictive value of pretreatment serum LDH, CA19-9, CRP and ALB levels for OS were evaluated, and the same as combination of the four factors. It was confirmed that serum LDH, CA19-9, CRP and ALB levels were independent prognostic factors for OS by multivariate analyses. The results of Kaplan-Meier analyses revealed that serum LDH, CA19-9, CRP, ALB levels as well as the combination of the four factors were correlated with OS. It's concluded that the combination of the pretreatment serum LDH, CA19-9, CRP and ALB levels is a prognostic factor for advanced PDAC patients.
\end{abstract}

Key words: Pancreatic cancer, Lactate Dehydrogenase, CA-19-9 Antigen, C-reactive protein, Albumin, Prognosis

\section{Introduction}

Pancreatic cancer is one of the most lethal malignant tumors, remaining the $4^{\text {th }}$ leading cause of cancer-related mortality[1, 2]. Most patients are diagnosed with advanced disease and resistant to chemoradiotherapy, consequently, the median OS of PDAC is less than 1 year and 5-year survival is approximately $5 \%$ [3].Therefore, it's urgent to identify biomarkers to predict patients' prognoses more precisely and guide better individual therapies.

Prior study has shown that cancer cells always synthesize abnormal serum enzymes[4], such as LDH, a key enzyme in glycolysis. Universally known, glycolysis is a distinctive metabolism feature in cancer cells. The strong relationship among serum LDH level, tumor burden[5] and patients' prognosis has been reported in colorectal cancer, non-Hodgkin lymphomas and renal cell carcinoma[6-8]. Serum CA19-9 is an essential biomarker in PDAC $[9,10]$, and widely used for predicting tumor progression $[9,11]$. Increasing evidences have suggested that persistent inflammation plays a vital role in carcinogenesis and prognosis[12, 13, 14]. Serum CRP is commonly used as an important biomarker for systemic inflammation and has been reported as a potential prognostic factor in various tumors[15, 16, 17]. As an important nutritional status index, several researches have proved the association between serum ALB level and survival in lung and ovarian cancers[18, 19]. 
Although the prognostic values of serum LDH, CA19-9, CRP and ALB levels have been confirmed in some reports, the debate continues about the validity in predicting prognosis by single parameter. So far, few studies investigated the survival model based on the combination of tumor metabolism, tumor biomarker, systemic inflammation and nutritional status in PDAC. Therefore, this retrospective study is aimed to investigate the prognostic value of the survival model combining the four biomarkers in advanced PDAC patients.

\section{Materials and Methods}

\section{Patients}

94 patients with locally advanced or metastatic PDAC receiving palliative chemotherapy in Shanghai General Hospital from 2009 to 2017 were recruited. All patients had baseline evaluation before treatment, including chest contrast enhanced computed tomography and abdominal contrast enhanced computed tomography or magnetic resonance. Eligibility criteria required individuals to have pathologically diagnoses of PDAC with the TNM

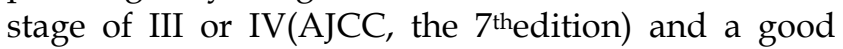
performance status( Karnofsky performance scores $\geq$ 80). Patients were previous treated with chemotherapy or radiotherapy were excluded. All patients were regularly followed-up on phone calls. Ethical approval was obtained by ethics committees of Shanghai General Hospital.

\section{Laboratory measurements}

Serum LDH, ALP, CA19-9, CEA, CRP and ALB levels were assayed during routine workups before treatment initiation. The cut-off value for the pre-treatment laboratory factors were defined as the ULN for LDH, ALP, CA19-9, CEA, and CRP, and the LLN for ALB.

\section{Study design and statistical analysis}

The primary aim of this retrospective study was to define a survival model based on tumor metabolism, tumor biomarker, systemic inflammation and nutritional status. All statistical analyses were performed using SPSS software (version20.0). Patients' characteristics were reported by descriptive statistics. The Pearson $\chi^{2}$ or Fisher's exact tests were used to compare clinicopathologic characteristics between groups, and non-parametric test was used to compare OS between groups. The HR was calculated using Cox proportional hazards regression model, by univariate and multivariate analysis respectively. The Kaplan-Meier method was used to estimate the survival curves with log-rank test assessing the significance. OS was defined as the time from the date of pathological diagnosis to death or the last follow-up visit. Statistical significance was defined with the $\mathrm{p}$ value $\leq 0.05$.

\section{Result}

\section{Patient characteristics}

94 patients with locally advanced or metastatic PDAC in Shanghai General Hospital from 2009 to 2017 were included in this study and the general characteristics are summarized in Table 1 . The median age at diagnosis was 61 years old (range from 34 to 86 years old) with 67 males $(71.3 \%)$. At treatment initiation, 75 patients $(79.8 \%)$ were in stage IV based on the TNM staging system, consistent with the number of patients with metastasis (75 patients had metastasis).Besides, 82 patients $(87.2 \%)$ had regional lymph node involvement. Following baseline evaluation, 71 patients $(75.5 \%)$ received gemcitabine based palliative chemotherapy, and the rest received other chemotherapy (5-Fu based chemotherapy or albumin-bound paclitaxel). The median OS was 8.0 months, and was significantly longer for patients with early stage, no-lymph nodes involvement, or no-distant metastasis $(\mathrm{p}=0.016, \mathrm{p}<0.001, \mathrm{p}=0.016$ ). The table illustrated that there were no significant differences in OS between different groups of clinicopathologic characteristics except stage, regional lymph nodes and metastasis.

Table 1 clinicopathologic characteristics of patients with advanced PDAC

\begin{tabular}{|c|c|c|c|c|}
\hline Characteristics & Number & Percentage(\%) & $\begin{array}{l}\text { Median } \\
\text { OS(month) }\end{array}$ & p-value \\
\hline Total & 94 & 100 & 8.0 & \\
\hline \multicolumn{5}{|l|}{ Age(years) } \\
\hline \multicolumn{5}{|l|}{ Median(range)61(34-86) } \\
\hline$<60$ & 41 & 43.6 & 8.0 & 0.985 \\
\hline$\geq 60$ & 53 & 56.4 & 8.0 & \\
\hline \multicolumn{5}{|l|}{ Gender } \\
\hline Male & 67 & 71.3 & 8.0 & 0.750 \\
\hline Female & 27 & 28.7 & 9.0 & \\
\hline \multicolumn{5}{|l|}{ Stage } \\
\hline III & 19 & 20.2 & 16.0 & 0.016 \\
\hline IV & 75 & 79.8 & 8.0 & \\
\hline \multicolumn{5}{|l|}{ Primary Tumor(T) } \\
\hline 2 & 5 & 5.3 & 10.0 & 0.919 \\
\hline $3 \& 4$ & 89 & 94.7 & 8.0 & \\
\hline \multicolumn{5}{|c|}{ Regional Lymph Nodes(N) } \\
\hline 0 & 12 & 12.8 & 20.5 & $<0.001$ \\
\hline 1 & 82 & 87.2 & 7.0 & \\
\hline \multicolumn{5}{|l|}{ Distant Metastasis(M) } \\
\hline 0 & 19 & 20.2 & 16.0 & 0.016 \\
\hline 1 & 75 & 79.8 & 8.0 & \\
\hline \multicolumn{5}{|l|}{ Treatment } \\
\hline $\begin{array}{l}\text { Gemcitabine based } \\
\text { chemotherapy }\end{array}$ & 71 & 75.5 & 8.0 & 0.888 \\
\hline Other chemotherapy & 23 & 24.5 & 8.0 & \\
\hline
\end{tabular}




\section{Independent prognostic factors for advanced PDAC}

To identify the risk factors linked to OS of patients with advanced PDAC, Cox proportional hazards regression model was used. As shown in Table 2, the significant prognostic factors for OS of patients with advanced PDAC were regional lymph nodes involvement $(\mathrm{HR}=3.337, \mathrm{p}=0.001)$, serum $\mathrm{LDH}$ $(\mathrm{HR}=4.915, \mathrm{p}<0.001), \quad$ ALP $\quad(\mathrm{HR}=3.081, \mathrm{p}<0.001)$, CA19-9 (HR=2.180, $\mathrm{p}=0.001), \quad \mathrm{CEA} \quad(\mathrm{HR}=2.586$, $\mathrm{p}=0.001), \quad \mathrm{CRP} \quad(\mathrm{HR}=3.429, \mathrm{p}<0.001) \quad$ and ALB $(\mathrm{HR}=1.736, \mathrm{P}=0.001)$ levels from univariate analysis, and they were significantly associated with poor OS. Then, the results obtained from the multivariate analysis of all the 13 factors revealed that regional lymph nodes involvement $(\mathrm{HR}=2.287, \mathrm{p}=0.034)$, serum LDH $(\mathrm{HR}=2.450, \mathrm{p}=0.004), \mathrm{CA19}-9(\mathrm{HR}=2.021$, $\mathrm{p}=0.05), \quad \mathrm{CRP} \quad(\mathrm{HR}=1.992, \quad \mathrm{p}=0.018)$ and ALB $(\mathrm{HR}=2.417, \quad \mathrm{P}=0.014)$ levels were identified as independent prognostic factors of OS in advanced PDAC patients, associating with poor prognosis (Table 2).

\section{Serum LDH and CA19-9 levels correlated with stage, regional lymph nodes and metastasis}

The correlations between different groups and clinicopathologic characteristics were evaluated using Pearson $\chi^{2}$ or Fisher's exact tests. The results, as shown in Table 3, late stage $(p=0.036, p=0.011)$, regional lymph nodes involvement $(\mathrm{p}=0.035,0.026)$ and distant metastasis $(p=0.036,0.011)$ were significantly correlated with high serum LDH and CA19-9 levels. However, no significant correlations were found between CRP and ALB subgroups and clinicopathologic characteristics.

Then, one elevation of serum LDH, CA19-9, CRP or the decrease of serum ALB levels was defined as a score of 1.Consequently, patients were divided into five groups with the scores of $0,1,2,3$ and 4 , respectively. However, as can be seen from table 3, no significant correlation was found between clinicapathologic characteristics and the scores.

Table 2 Univariate and multivariate analysis of prognostic factors

\begin{tabular}{|c|c|c|c|}
\hline Variable & Patients(n) & $\mathrm{HR}(95 \% \mathrm{CI})$ & P-value \\
\hline \multicolumn{4}{|l|}{ Univariate analysis } \\
\hline $\begin{array}{l}\text { Age } \\
(<60 \text { vs. } \geq 60)\end{array}$ & 41 vs. 53 & $0.929(0.609-1.416)$ & 0.731 \\
\hline $\begin{array}{l}\text { Gender } \\
\text { (male vs. female) }\end{array}$ & 67 vs. 27 & $0.719(0.443-1.169)$ & 0.184 \\
\hline $\begin{array}{l}\text { Stage } \\
\text { (III vs. IV) }\end{array}$ & 19 vs. 75 & $1.654(0.971-2.819)$ & 0.064 \\
\hline $\begin{array}{l}\text { Primary Tumor }(\mathrm{T}) \\
(2 \text { vs. } 3 \text { and } 4)\end{array}$ & 5 vs. 89 & $0.876(0.354-2.165)$ & 0.774 \\
\hline $\begin{array}{l}\text { Regional Lymph } \operatorname{Nodes}(\mathrm{N}) \\
(0 \text { vs. } 1)\end{array}$ & 12 vs. 82 & $3.337(1.645-6.770)$ & 0.001 \\
\hline $\begin{array}{l}\text { Distant Metastasis }(\mathrm{M}) \\
(0 \mathrm{vs.})\end{array}$ & 19 vs. 75 & $1.654(0.971-2.819)$ & 0.064 \\
\hline $\begin{array}{l}\text { Regiment } \\
\text { (Gemcitabine vs. Others) }\end{array}$ & 71 vs. 23 & $1.213(0.751-1.958)$ & 0.430 \\
\hline $\begin{array}{l}\text { LDH } \\
(\leq \mathrm{ULN} \text { vs. }>\mathrm{ULN})\end{array}$ & 71 vs. 23 & $4.915(2.907-8.311)$ & $<0.001$ \\
\hline $\begin{array}{l}\text { ALP } \\
(\leq \mathrm{ULR} \text { vs. }>\text { ULN) }\end{array}$ & 46 vs. 48 & $3.081(1.946-4.878)$ & $<0.001$ \\
\hline $\begin{array}{l}\text { CA19-9 } \\
(\leq \text { ULN vs. }>\text { ULN })\end{array}$ & 40 vs. 54 & $2.180(1.387-3.427)$ & 0.001 \\
\hline $\begin{array}{l}\text { CEA } \\
(\leq \mathrm{ULN} \text { vs. }>\mathrm{ULN})\end{array}$ & 25 vs. 69 & $2.689(1.534-4.714)$ & 0.001 \\
\hline $\begin{array}{l}\text { CRP } \\
(\leq \mathrm{ULN} \text { vs. }>\mathrm{ULN})\end{array}$ & 52 vs. 42 & $3.429(2.165-5.429)$ & $<0.001$ \\
\hline $\begin{array}{l}\text { Albumin } \\
(\geq \text { LLN vs. }<\text { LLN })\end{array}$ & 83 vs. 11 & $1.736(1.248-2.414)$ & 0.001 \\
\hline Multivariate analysis & & & \\
\hline $\begin{array}{l}\text { Regional Lymph } \operatorname{Nodes}(\mathrm{N}) \\
(0 \text { vs. } 1)\end{array}$ & 12 vs. 82 & $2.474(1.176-5.203)$ & 0.017 \\
\hline $\begin{array}{l}\text { LDH } \\
(\leq \mathrm{ULN} \text { vs. }>\text { ULN })\end{array}$ & 71 vs. 23 & $3.181(1.735-5.833)$ & $<0.001$ \\
\hline $\begin{array}{l}\text { CA19-9 } \\
(\leq \text { ULN vs. }>\text { ULN })\end{array}$ & 40 vs. 54 & $2.020(1.235-3.302)$ & 0.005 \\
\hline $\begin{array}{l}\text { CRP } \\
(\leq \mathrm{ULN} \text { vs. }>\text { ULN })\end{array}$ & 52 vs. 42 & 2.214(1.304-3.758) & 0.003 \\
\hline $\begin{array}{l}\text { Albumin } \\
(\geq \text { LLN vs. }<\text { LLN) }\end{array}$ & 83 vs. 11 & $2.417(1.194-4.890)$ & 0.014 \\
\hline
\end{tabular}

Table 3 Correlation between LDH/CA19-9/CRP/ALB and clinicopathologic characteristics

\begin{tabular}{|c|c|c|c|c|c|c|c|c|c|c|c|c|c|c|c|c|c|c|c|}
\hline \multirow[t]{2}{*}{ Characteristics } & \multirow[t]{2}{*}{ Value } & \multicolumn{3}{|c|}{ Serum LDH Level } & \multicolumn{3}{|c|}{ Serum CA19-9 Level } & \multicolumn{3}{|c|}{ Serum CRP Level } & \multicolumn{3}{|c|}{ Serum ALB Level } & \multicolumn{6}{|c|}{ Combination of 4 factors } \\
\hline & & $\leq \mathrm{ULN}$ & $>\mathrm{ULN}$ & p-value & $\leq \mathrm{ULN}$ & $>\mathrm{ULN}$ & p-value & $\leq \mathrm{ULN}$ & $>\mathrm{ULN}$ & p-value & $\geq \mathrm{LLN}$ & $<\mathrm{LLN}$ & p-value & 0 & 1 & 2 & 3 & 4 & p-value \\
\hline \multirow[t]{2}{*}{ Age(years) } & $<60$ & 30 & 11 & 0.640 & 17 & 24 & 0.851 & 21 & 20 & 0.482 & 36 & 5 & 0.896 & 10 & 11 & 12 & 7 & 1 & 0.389 \\
\hline & $\geq 60$ & 41 & 12 & & 23 & 30 & & 31 & 22 & & 47 & 5 & & 14 & 21 & 8 & 7 & 3 & \\
\hline \multirow[t]{2}{*}{ Gender } & Male & 49 & 18 & 0.394 & 28 & 39 & 0.814 & 37 & 30 & 0.977 & 61 & 6 & 0.192 & 16 & 24 & 15 & 9 & 3 & 0.915 \\
\hline & Female & 22 & 5 & & 12 & 15 & & 15 & 12 & & 22 & 5 & & 8 & 8 & 5 & 5 & 1 & \\
\hline \multirow[t]{2}{*}{ Stage } & III & 18 & 1 & 0.036 & 13 & 6 & 0.011 & 13 & 6 & 0.198 & 17 & 2 & 1.000 & 8 & 8 & 2 & 1 & 0 & 0.146 \\
\hline & IV & 53 & 22 & & 27 & 48 & & 39 & 36 & & 66 & 9 & & 16 & 24 & 18 & 13 & 4 & \\
\hline Primary & 2 & 3 & 2 & 0.593 & 1 & 4 & 0.390 & 3 & 2 & 1.000 & 5 & 0 & 1.000 & 1 & 2 & 0 & 2 & 0 & 0.448 \\
\hline Tumor(T) & 3 and 4 & 68 & 21 & & 39 & 50 & & 49 & 40 & & 78 & 11 & & 23 & 30 & 20 & 12 & 4 & \\
\hline Regional & 0 & 12 & 0 & 0.035 & 9 & 3 & 0.026 & 8 & 4 & 0.538 & 11 & 1 & 1.000 & 6 & 4 & 2 & 0 & 0 & 0.199 \\
\hline $\begin{array}{l}\text { Lymph } \\
\text { Nodes(N) }\end{array}$ & 1 & 59 & 23 & & 31 & 51 & & 44 & 38 & & 72 & 10 & & 18 & 28 & 18 & 14 & 4 & \\
\hline Distant & 0 & 18 & 1 & 0.036 & 13 & 6 & 0.011 & 13 & 6 & 0.198 & 17 & 2 & 1.000 & 8 & 8 & 2 & 1 & 0 & 0.146 \\
\hline Metastasis(M) & 1 & 53 & 22 & & 27 & 48 & & 39 & 36 & & 66 & 9 & & 16 & 24 & 18 & 13 & 4 & \\
\hline \multirow[t]{2}{*}{ Treatment } & Gemcitabine & 55 & 16 & 0.444 & 29 & 42 & 0.556 & 40 & 31 & 0.727 & 64 & 7 & 0.454 & 18 & 26 & 14 & 10 & 3 & 0.905 \\
\hline & Others & 16 & 7 & & 11 & 12 & & 12 & 11 & & 19 & 4 & & 6 & 6 & 6 & 4 & 1 & \\
\hline
\end{tabular}


A

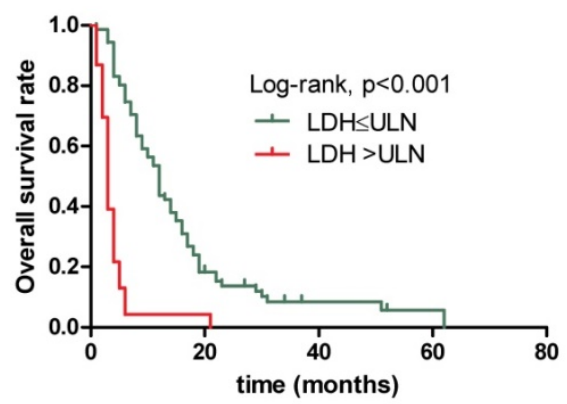

C

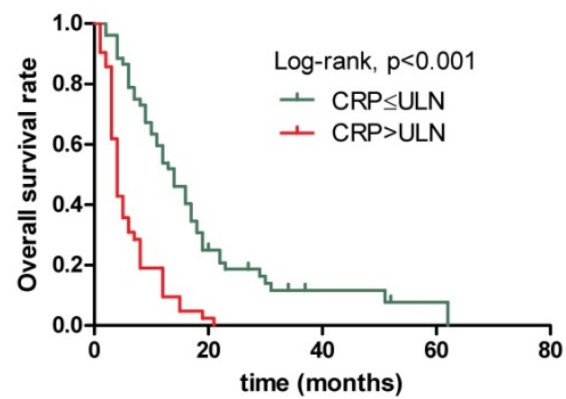

B

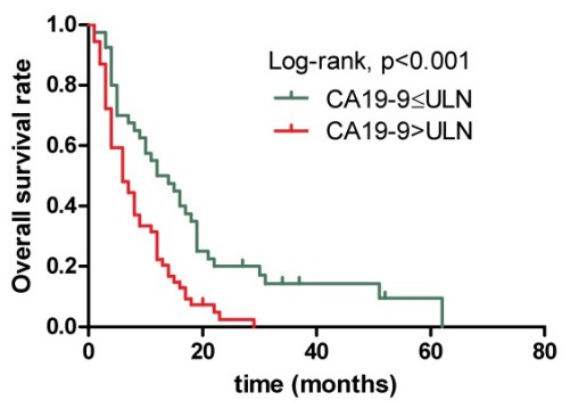

D

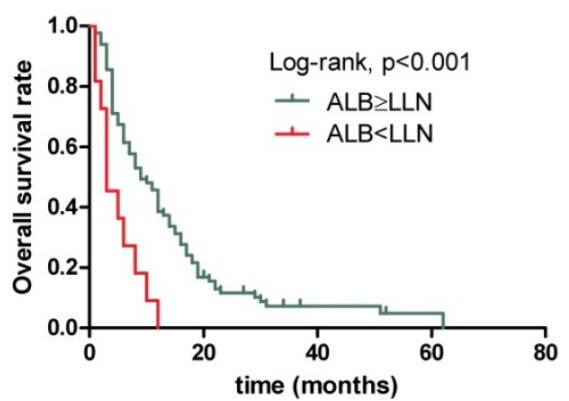

Figure 1. Kaplan-Meier overall survival curves for 94 patients with locally advanced and metastatic PDAC stratified by (A) pretreatment serum LDH level, (B) pretreatment serum CA19-9 level, (C) pretreatment serum CRP level and (D) pretreatment ALB level.

\section{Elevated serum LDH, CA19-9 and CRP levels, decreased serum ALB level and high combination score associated with poor prognosis}

The results obtained from non-parametric test revealed that patients with low serum LDH, CA19-9 and CRP levels and high serum ALB level tended to have longer OS (12.0 vs. 3.0 months, $\mathrm{p}<0.001,13.0$ vs. 6.0 months, $\mathrm{p}=0.001$ and 14.0 vs. 4.0 months, $\mathrm{p}<0.001,9.0$ vs. 3.0 months, $\mathrm{p}=0.004$, respectively). Further, when combining the four prognostic factors, the patients with higher scores had shorter OS (18.5 vs. 10.5 vs. 6.0 vs. 3.0 vs. 2.0 months, $p<0.001)$. Next, Figure 1 presents similar results using Kaplan-Meier method. The strong correlations were observed between subgroups of serum LDH, CA19-9, CRP and ALB levels and OS from survival curves $(p<0.001$, $p<0.001, p<0.001$ and $p<0.001$, respectively). In addition, the prognostic role of combination of LDG, CA19-9, CRP and ALB was observed and robust correlation with OS is presented in Figure $2(p<0.001)$. The results suggested that patients with lower serum LDH, CA19-9 and CRP levels, higher serum ALB level and lower combination score were associated with better prognosis. However, no differences were observed in subgroups of CA19-9 and CRP when patients with high LDH level, LDH and ALB with normal CA19-9 level, CA19-9 and ALB with high CRP level, and CRP with low ALB level (Supplementary).

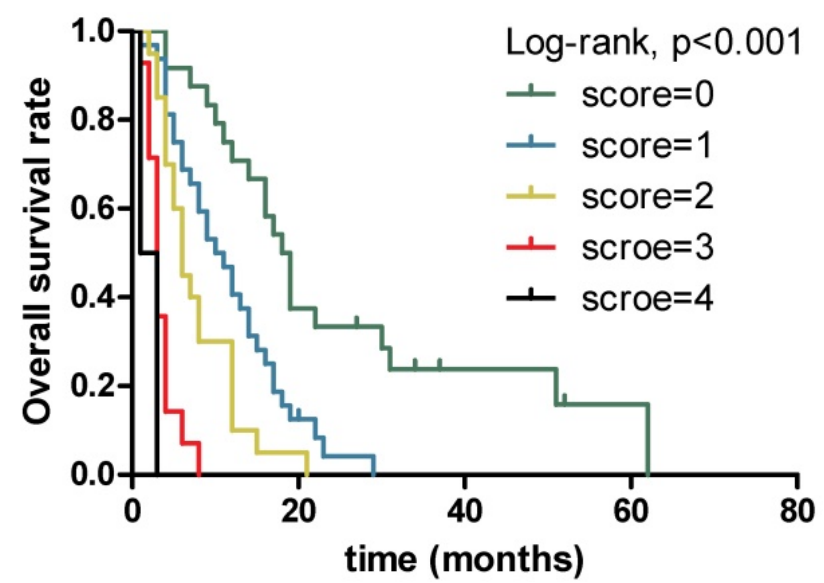

Figure 2. Kaplan-Meier overall survival curves for 94 patients with locally advanced and metastatic PDAC stratified by different scores combining serum LDH, CA19-9, CRP and ALB levels.

\section{Discussion}

In this retrospective study, we investigated the clinical significance and prognostic role of 7 clinical variables and 6 serum biochemical markers in 94 
patients with locally advanced and metastatic PDAC. All the parameters were included in the Cox proportional hazards regression model in both univariate and multivariate analysis. The 7 clinical variables are important clinical characteristics for PDAC. Widely acknowledged, stage, primary tumor, lymph node involvement and metastasis may be associated with prognosis. In the 7 clinical variables, the stage of primary tumor and the treatment regiments were divided into 2 parts, despite they have 3 parts. The reasons lay on the fact whether the tumor is easy for resection and gemcitabine is the first-line chemotherapy for patients. And we also analyzed the data with the 2 factor divided into 3 parts, the results were the same with that they were divided into 2 parts. The 6 serum biochemical markers are common laboratory tests which can be easily obtained for patients. Serum LDH and ALP levels are important metabolism markers and they have been proved to associate with the prognosis of PDAC[20, 21]. Serum CEA and CA19-9 levels are the most widely used biomarkers for diagnosis and prediction of prognosis in PDAC[22, 23]. As biomarkers for systemic inflammatory response and nutrition status, several studies have revealed that they are prognostic factors of survival in patients with PDAC[24, 25]. The results of multivariate analysis suggested that regional lymph nodes involvement was the only clinical factors associated with OS. Surprisingly, the stage, size of primary tumor and distance metastasis were not significantly associated with OS, probably due to the reasons that patients included were with late stage and all patients with $\mathrm{T} 2$ had distance metastasis indicating worse biological characteristics with metastasizing in early stage. As for serum biochemical markers, serum LDH, CA19-9, CRP and ALB levels were associated with OS. Serum ALP and CEA levels were excluded from the survival model after multivariate analysis, meaning that they are not independent factors for survival. A possible explanation for this is that ALP and CEA correlate with LDH and CA19-9, respectively, and they probably influence the prognosis through LDH and CA19-9. Patients with high serum LDH, CA19-9, and CRP levels or low serum ALB level had shorter OS. The four serum biochemical markers are routinely tested and the certain levels can be easily obtained in clinical practice, however, inaccuracy might exist on the evaluation of regiment lymph nodes involvement, owing to the subjective factors in imaging diagnosis. Accordingly, serum LDH, CA19-9, CRP and ALB levels are included in our survival model.

Warburg's report revealed that, anaerobic glycolysis is preferred by tumor cells, despite of the sufficiency of oxygen[26]. LDH, converting pyruvate to lactate, is a key enzyme in metabolism of tumor cells[27]. It has been reported that there are strong relationship between $\mathrm{LDH}$ and hypoxia microenvironment, commonly seen in PDAC, because oxygen and nutrients are obstructed by desmoplastic stroma. Through hypoxia inducible factors (HIFs), $\mathrm{LDH}$ and hypoxia microenvironment are associated with metastasis and worse prognosis in several tumors[28-30]. These metabolic changes in tumor progression cause changes of serum enzymes, such as serum LDH and ALP levels. High serum LDH level has been proved to be correlated with short OS in nasopharyngeal carcinoma[31]. Therefore, we investigated the relationship between serum $\mathrm{LDH}$ level and OS in patients with advanced PDAC. And we found high serum LDH level was significantly corrected with short OS. In patients with advanced PDAC, CA19-9 is the most commonly used biomarker in predicting prognosis[32, 33]. Our findings confirmed that serum CA19-9 level had strong association with the prognosis in advanced PDAC patients and consistent with the results universally known, patients with low serum CA19-9 level had better prognosis, .

Recently, it has reported that inflammation tumor microenvironment is important for tumor proliferation and survival[34, 35]. Besides, systemic inflammation also indicates poor prognosis[34, 35]. Serum CRP level, a biomarker of systemic inflammation, whose elevation correlates with poor prognosis is proved in colorectal cancer and pancreatic cancer[15, 36-39]. But, inversed results have been found in some studies[40, 41]. In this research, we found that high serum CRP level was a risk factor for prognosis. Low serum ALB level, indicating malnutrition, commonly occurs in patients with advanced PDAC. Our research demonstrated that lower serum ALB level was associated with poorer prognosis and shorter OS.

However, single biomarker cannot predict the certain patient's prognosis precisely, for instance, PDAC patients who lake of Lewis antigen will not suffer elevation of CA19-9, a part of Lewis antigen. Additionally, CA19-9 will elevate transitory if patients have systemic inflammation. So, we analyzed the rest 3 factors in 2 subgroups of each biomarker. We found that when patients with high serum LDH level, there were no significance for CA19-9 and CRP as biomarker, similar results had found in LDH and ALB in patients with normal CA19-9 level, CA19-9 and ALB with high CRP level and CRP with low ALB level. To eliminate the inaccuracy in single biomarker for predicting patients' prognosis, we combined the 4 factors presenting tumor metabolism, tumor biomarker, systemic inflammation and nutritional 
status to predict a certain patient's prognosis more precisely, by one positive value counting 1 point. The investigation showed that patients with higher score suffered significantly shorter OS. Because this score system evaluates patients' tumor metabolism, tumor marker, inflammation and nutrition comprehensively, we might predict the prognosis precisely and make more suitable treatment and evaluation plan for patients more likely to suffer bad prognosis. Nevertheless, the first limitation of this retrospect single-center study is the comparatively small sample size, leading to the fact that we could not divide the patients into training cohort and validation cohort. Besides, as a single-center study, selection bias is hard to be avoided. Finally, it's difficult to collect all the information completely in a retrospect study. In the future, we will design a prospective multi-center study and validate the results.

\section{Conclusion}

Our study confirmed the crucial role of serum LDH, CA19-9, CRP and ALB as biomarkers in predicting prognosis in patients with advanced PDAC. To precisely determine patients' prognosis, the combination of 4 factors, considered tumor metabolism, tumor biomarker, systemic inflammation and nutritional status comprehensively, facilitates precision management and prolong the survival in patients with advanced PDAC. Our future work will focus on expand the number of patients and validate its accuracy.

\section{Abbreviations}

LDH: lactate dehydrogenase; CA19-9: carbohydrate antigen 19-9; CRP: C-reactive protein; ALB: albumin; PDAC: pancreatic ductal adenocarcinoma; OS: overall survival; ALP: alkaline phosphatase; CEA: carcinoembryonic antigen; ULN: upper limit of normal; LLN: lower limit of normal; HR: hazard ratio.

\section{Supplementary Material}

Supplementary figures.

http://www.jcancer.org/v09p1301s1.pdf

\section{Acknowledgment}

This work was supported by the National Natural Science Foundation of China (Grant NO. 81502017, 81502018, 81572 315).

\section{Competing Interests}

The authors have declared that no competing interest exists.

\section{References}

1. Jemal A, Siegel R, Xu J, et al. Cancer statistics, 2010. CA Cancer J Clin. 2010; 60: 277-300.

2. Jemal A, Bray F, Center MM, et al. Global cancer statistics. CA Cancer J Clin. 2011; 61: 69-90.

3. Biankin AV, Waddell N, Kassahn KS, et al. Pancreatic cancer genomes reveal aberrations in axon guidance pathway genes. Nature. 2012; 491: 399-405.

4. Ferreira LM, Hebrant A, Dumont JE. Metabolic reprogramming of the tumor. Oncogene. 2012; 31: 3999-4011.

5. Tas F, Aykan F, Alici S, et al. Prognostic factors in pancreatic carcinoma: serum LDH levels predict survival in metastatic disease. Am J Clin Oncol. 2001; 24: 547-50.

6. Scartozzi M, Giampieri R, Maccaroni E, et al. Pre-treatment lactate dehydrogenase levels as predictor of efficacy of first-line bevacizumab-based therapy in metastatic colorectal cancer patients. Br J Cancer. 2012; 106: 799-804.

7. Ferraris AM, Giuntini P, Gaetani GF. Serum lactic dehydrogenase as a prognostic tool for non-Hodgkin lymphomas. Blood. 1979; 54: 928-32.

8. Armstrong AJ, George DJ, Halabi S. Serum lactate dehydrogenase predicts for overall survival benefit in patients with metastatic renal cell carcinoma treated with inhibition of mammalian target of rapamycin. J Clin Oncol. 2012; 30: 3402-7.

9. Boeck $\mathrm{S}$, Stieber $\mathrm{P}$, Holdenrieder $\mathrm{S}$, et al. Prognostic and therapeutic significance of carbohydrate antigen 19-9 as tumor marker in patients with pancreatic cancer. Oncology. 2006; 70: 255-64.

10. Asaoka T, Miyamoto A, Maeda S, et al. Prognostic impact of preoperative NLR and CA19-9 in pancreatic cancer. Pancreatology. 2016; 16: 434-40.

11. Bauer TM, El-Rayes BF, Li X, et al. Carbohydrate antigen 19-9 is a prognostic and predictive biomarker in patients with advanced pancreatic cancer who receive gemcitabine-containing chemotherapy: a pooled analysis of 6 prospective trials. Cancer. 2013; 119: 285-92.

12. Coussens LM, Werb Z. Inflammation and cancer. Nature. 2002; 420: 860-7.

13. Mantovani A, Allavena P, Sica A, et al. Cancer-related inflammation. Nature. 2008; 454: 436-44.

14. Lee JM, Lee HS, Hyun JJ, et al. Prognostic value of inflammation-based markers in patients with pancreatic cancer administered gemcitabine and erlotinib. World J Gastrointest Oncol. 2016; 8: 555-62.

15. Erlinger TP, Platz EA, Rifai N, et al. C-reactive protein and the risk of incident colorectal cancer. Jama. 2004; 291: 585-90.

16. Jamieson NB, Glen P, McMillan DC, et al. Systemic inflammatory response predicts outcome in patients undergoing resection for ductal adenocarcinoma head of pancreas. Br J Cancer. 2005; 92: 21-3.

17. Wu M, Guo J, Guo L, et al. The C-reactive protein/albumin ratio predicts overall survival of patients with advanced pancreatic cancer. Tumour Biol. 2016; 37: 12525-33.

18. Jin Y, Zhao L, Peng F. Prognostic impact of serum albumin levels on the recurrence of stage I non-small cell lung cancer. Clinics (Sao Paulo). 2013; 68: 686-93.

19. Ataseven B, du Bois A, Reinthaller A, et al. Pre-operative serum albumin is associated with post-operative complication rate and overall survival in patients with epithelial ovarian cancer undergoing cytoreductive surgery. Gynecol Oncol. 2015; 138: 560-5.

20. Ji F, Fu SJ, Guo ZY, et al. Prognostic value of combined preoperative lactate dehydrogenase and alkaline phosphatase levels in patients with resectable pancreatic ductal adenocarcinoma. Medicine(Baltimore). 2016; 95: e4065.

21. Faloppi L, Bianconi M, Giampieri R, et al. The value of lactate dehydrogenase serum levels as a prognostic and predictive factor for advanced pancreatic cancer patients receiving sorafenib. Oncotarget. 2015; 6: 35087-94.

22. Tas F, Karabulut S, Ciftci R, et al. Serum levels of LDH, CEA, and CA19-9 have prognostic roles on survival in patients with metastatic pancreatic cancer receiving gemcitabine-based chemotherapy. Cancer Chemother Pharmacol. 2014; 73: 1163-71.

23. Haas M, Heinemann V, Kullmann F, et al. Prognostic value of CA 19-9, CEA, CRP, LDH and bilirubin levels in locally advanced and metastatic pancreatic cancer: results from a multicenter, pooled analysis of patients receiving palliative chemotherapy. J Cancer Res Clin Oncol. 2013; 139: 681-9.

24. Yu SL, Xu LT, Qi Q, et al. Serum lactate dehydrogenase predicts prognosis and correlates with systemic inflammatory response in patients with advanced pancreatic cancer after gemcitabine-based chemotherapy. Sci Rep. 2017; 7: 45194.

25. Nakano $\mathrm{Y}$, Kitago M, Shinoda M, et al. Clinical predictive factors of long-term survival after curative resection of pancreatic cancer: a retrospective study. Cancer Med. 2017; 6: 2278-86.

26. Warburg O. On the origin of cancer cells. Science. 1956; 123: 309-14.

27. Zhou W, Capello M, Fredolini C, et al. MS analysis reveals O-methylation of L-lactate dehydrogenase from pancreatic ductal adenocarcinoma cells. Electrophoresis. 2012; 33: 1850-4.

28. Maftouh M, Avan A, Sciarrillo R, et al. Synergistic interaction of novel lactate dehydrogenase inhibitors with gemcitabine against pancreatic cancer cells in hypoxia. Br J Cancer. 2014; 110: 172-82.

29. Lu H, Forbes RA, Verma A. Hypoxia-inducible factor 1 activation by aerobic glycolysis implicates the Warburg effect in carcinogenesis. J Bio Chem. 2002; 277: 23111-5.

30. Lu X, Kang Y. Hypoxia and hypoxia-inducible factors: master regulators of metastasis. Clin Cancer Res. 2010; 16: 5928-35. 
31. Li G, Gao J, Tao YL, et al. Increased pretreatment levels of serum LDH and ALP as poor prognostic factors for nasopharyngeal carcinoma. Chin J Cancer. 2012; 31: 197-206.

32. Qi Q, Geng Y, Sun M, et al. Clinical implications of systemic inflammatory response markers as independent prognostic factors for advanced pancreatic cancer. Pancreatology. 2015; 15: 145-50.

33. Tas F, Karabulut S, Ciftci R, et al. Serum levels of LDH, CEA, and CA19-9 have prognostic roles on survival in patients with metastatic pancreatic cancer receiving gemcitabine-based chemotherapy. Cancer chemother Pharmacol. 2014; 73: 1163-71.

34. Whiteside TL. The tumor microenvironment and its role in promoting tumor growth. Oncogene. 2008; 27: 5904-12.

35. Diakos CI, Charles KA, McMillan DC, et al. Cancer-related inflammation and treatment effectiveness. Lancet Oncol. 2014; 15: e493-503.

36. Sanjay P, de Figueiredo RS, Leaver H, et al. Preoperative serum C-reactive protein levels and post-operative lymph node ratio are important predictors of survival after pancreaticoduodenectomy for pancreatic ductal adenocarcinoma. Jop. 2012; 13: 199-204.

37. Gunter MJ, Stolzenberg-Solomon R, Cross AJ, et al. A prospective study of serum C-reactive protein and colorectal cancer risk in men. Cancer Res. 2006; 66: 2483-7.

38. Falconer JS, Fearon KC, Ross JA, et al. Acute-phase protein response and survival duration of patients with pancreatic cancer. Cancer. 1995; 75: 2077-82.

39. Ueno $\mathrm{H}$, Okada S, Okusaka $\mathrm{T}$, et al. Prognostic factors in patients with metastatic pancreatic adenocarcinoma receiving systemic chemotherapy. Oncology. 2000; 59: 296-301.

40. Ognjanovic S, Yamamoto J, Saltzman B, et al. Serum CRP and IL-6, genetic variants and risk of colorectal adenoma in a multiethnic population. Cancer Causes Control. 2010; 21: 1131-8.

41. Zhang SM, Buring JE, Lee IM, et al. C-reactive protein levels are not associated with increased risk for colorectal cancer in women. Ann Intern Med. 2005; 142: 425-32. 